\title{
Erratum to: The John Charnley Award: A Study of Implant Failure in Metal-on-Metal Surface Arthroplasties
}

Pat Campbell PhD, Paul E. Beaulé MD,

Edward Ebramzadeh PhD, Michel J. Le Duff MA,

Koen De Smet MD, Zhen Lu PhD, Harlan C. Amstutz MD

Published online: 26 July 2011

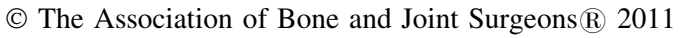

\section{Erratum to: Clin Orthop Relat Res \\ DOI 10.1097/01.blo.0000238777.34939.82}

In the original version of this article, which appeared in Volume 453, pages 35-46 (DOI 10.1097/01.blo.0000238777. 34939.82), the author listed as "Michel LeDuff, MA" should be listed as "Michel J. Le Duff, MA".

P. Campbell, M. J. Le Duff, H. C. Amstutz

Joint Replacement Institute at Orthopaedics Hospital,

Los Angeles, CA, USA

P. Campbell, E. Ebramzadeh, Z. Lu

UCLA David Geffen School of Medicine,

Los Angeles, CA, USA

P. Campbell, E. Ebramzadeh, Z. Lu

J. Vernon Luck Research Center at Orthopaedics Hospital,

Los Angeles, CA, USA

P. E. Beaulé

Division of Orthopedic Surgery, University of Ottawa,

Ottawa, Canada

K. De Smet

ANCA Clinic, Heusden, Belgium

P. Campbell $(\bowtie)$

Orthopaedic Hospital, 2400 S. Flower Street,

Los Angeles, CA 90007, USA

e-mail: pcampbell@laoh.ucla.edu 